\title{
Answers to Two Questions on the DP Color Function
}

\author{
Jeffrey A. Mudrock \\ Department of Mathematics \\ College of Lake County \\ Grayslake, IL, U.S.A.
}

\author{
Seth Thomason \\ Department of Mathematics \\ College of Lake County \\ Grayslake, IL, U.S.A.
}

jmudrock@clcillinois.edu

Submitted: Sep 15, 2020; Accepted: Apr 26, 2021; Published: May 21, 2021

(C) The authors. Released under the CC BY-ND license (International 4.0).

\begin{abstract}
DP-coloring is a generalization of list coloring that was introduced in 2015 by Dvořák and Postle. The chromatic polynomial of a graph is a notion that has been extensively studied since the early 20 th century. The chromatic polynomial of graph $G$ is denoted $P(G, m)$, and it is equal to the number of proper $m$-colorings of $G$. In 2019, Kaul and Mudrock introduced an analogue of the chromatic polynomial for DP-coloring; specifically, the DP color function of graph $G$ is denoted $P_{D P}(G, m)$. For vertex disjoint graphs $G$ and $H$, suppose $G \vee H$ denotes the join of $G$ and $H$. Two fundamental questions posed by Kaul and Mudrock are: (1) For any graph $G$ with $n$ vertices, is it the case that $P(G, m)-P_{D P}(G, m)=O\left(m^{n-3}\right)$ as $m \rightarrow \infty$ ? and (2) For every graph $G$, does there exist $p, N \in \mathbb{N}$ such that $P_{D P}\left(K_{p} \vee G, m\right)=P\left(K_{p} \vee G, m\right)$ whenever $m \geqslant N$ ? We show that the answer to both these questions is yes. In fact, we show the answer to (2) is yes even if we require $p=1$.
\end{abstract}

Mathematics Subject Classifications: 05C15, 05C30, 05C69

\section{Introduction}

In this note all graphs are nonempty, finite, simple graphs unless otherwise noted. Generally speaking we follow West [31] for terminology and notation. The set of natural numbers is $\mathbb{N}=\{1,2,3, \ldots\}$. For $m \in \mathbb{N}$, we write $[m]$ for the set $\{1, \ldots, m\}$. Given a set $A, \mathcal{P}(A)$ is the power set of $A$. If $G$ is a graph and $S, U \subseteq V(G)$, we use $G[S]$ for the subgraph of $G$ induced by $S$, and we use $E_{G}(S, U)$ for the set consisting of all the edges in $E(G)$ that have at least one endpoint in $S$ and at least one endpoint in $U$. If an edge in $E(G)$ connects the vertices $u$ and $v$, the edge can be represented by $u v$ or $v u$. If $G$ and $H$ are vertex disjoint graphs, we write $G \vee H$ for the join of $G$ and $H$. The cone of graph $G$ is $K_{1} \vee G$. 


\subsection{List Coloring and DP-Coloring}

In the classical vertex coloring problem we wish to color the vertices of a graph $G$ with up to $m$ colors from $[m]$ so that adjacent vertices receive different colors, a so-called proper $m$-coloring. The chromatic number of a graph $G$, denoted $\chi(G)$, is the smallest $m$ such that $G$ has a proper $m$-coloring. List coloring, a well-known variation on classical vertex coloring, was introduced independently by Vizing [29] and Erdős, Rubin, and Taylor [12] in the 1970s. For list coloring, we associate a list assignment $L$ with a graph $G$ such that each vertex $v \in V(G)$ is assigned a list of colors $L(v)$ (we say $L$ is a list assignment for $G)$. Then, $G$ is $L$-colorable if there exists a proper coloring $f$ of $G$ such that $f(v) \in L(v)$ for each $v \in V(G)$ (we refer to $f$ as a proper $L$-coloring of $G$ ). A list assignment $L$ is called a $k$-assignment for $G$ if $|L(v)|=k$ for each $v \in V(G)$. The list chromatic number of a graph $G$, denoted $\chi_{\ell}(G)$, is the smallest $k$ such that $G$ is $L$-colorable whenever $L$ is a $k$-assignment for $G$. We say $G$ is $k$-choosable if $k \geqslant \chi_{\ell}(G)$. Since $G$ must be $L$-colorable whenever $L$ is a $\chi_{\ell}(G)$-assignment for $G$ that assigns the same list of colors to each element in $V(G)$, it is clear that $\chi(G) \leqslant \chi_{\ell}(G)$. This inequality may be strict since it is known that there are bipartite graphs with arbitrarily large list chromatic number (see [12]).

In 2015, Dvořák and Postle [11] introduced a generalization of list coloring called DPcoloring (they called it correspondence coloring) in order to prove that every planar graph without cycles of lengths 4 to 8 is 3 -choosable. DP-coloring has been extensively studied over the past 5 years (see e.g., $[3,4,5,6,7,8,16,17,18,19,22,23,25,26]$ ). Intuitively, DP-coloring is a variation on list coloring where each vertex in the graph still gets a list of colors, but identification of which colors are different can change from edge to edge. Following [7], we now give the formal definition. Suppose $G$ is a graph. A cover of $G$ is a pair $\mathcal{H}=(L, H)$ consisting of a graph $H$ and a function $L: V(G) \rightarrow \mathcal{P}(V(H))$ satisfying the following four requirements:

(1) the set $\{L(u): u \in V(G)\}$ is a partition of $V(H)$ of size $|V(G)|$;

(2) for every $u \in V(G)$, the graph $H[L(u)]$ is complete;

(3) if $E_{H}(L(u), L(v))$ is nonempty, then $u=v$ or $u v \in E(G)$;

(4) if $u v \in E(G)$, then $E_{H}(L(u), L(v))$ is a matching (the matching may be empty).

Suppose $\mathcal{H}=(L, H)$ is a cover of $G$. We refer to the edges of $H$ connecting distinct parts of the partition $\{L(v): v \in V(G)\}$ as cross-edges. An $\mathcal{H}$-coloring of $G$ is an independent set in $H$ of size $|V(G)|$. It is immediately clear that an independent set $I \subseteq V(H)$ is an $\mathcal{H}$-coloring of $G$ if and only if $|I \cap L(u)|=1$ for each $u \in V(G)$. We say $\mathcal{H}$ is $m$-fold if $|L(u)|=m$ for each $u \in V(G)$. The DP-chromatic number of $G, \chi_{D P}(G)$, is the smallest $m \in \mathbb{N}$ such that $G$ has an $\mathcal{H}$-coloring whenever $\mathcal{H}$ is an $m$-fold cover of $G$.

Suppose $\mathcal{H}=(L, H)$ is an $m$-fold cover of $G$. We say that $\mathcal{H}$ has a canonical labeling if it is possible to name the vertices of $H$ so that $L(u)=\{(u, j): j \in[m]\}$ and $(u, j)(v, j) \in$ $E(H)$ for each $j \in[m]$ whenever $u v \in E(G) .{ }^{1}$ Clearly, when $\mathcal{H}$ has a canonical labeling, $G$

\footnotetext{
${ }^{1}$ When $\mathcal{H}=(L, H)$ has a canonical labeling, we refer to the vertices of $H$ using this naming scheme.
} 
has an $\mathcal{H}$-coloring if and only if $G$ has a proper $m$-coloring. Also, given an $m$-assignment, $L$, for a graph $G$, it is easy to construct an $m$-fold cover $\mathcal{H}^{\prime}$ of $G$ such that $G$ has an $\mathcal{H}^{\prime}$ coloring if and only if $G$ has a proper $L$-coloring (see [7]). It follows that $\chi(G) \leqslant \chi_{\ell}(G) \leqslant$ $\chi_{D P}(G)$. The second inequality may be strict since it is easy to prove that $\chi_{D P}\left(C_{n}\right)=3$ whenever $n \geqslant 3$, but the list chromatic number of any even cycle is 2 (see [7] and [12]).

In some instances DP-coloring behaves similar to list coloring, but there are some interesting differences. Molloy [25] has shown that Kahn's [14] result that the list edgechromatic number of a simple graph asymptotically equals the edge-chromatic number holds for DP-coloring as well. Thomassen [27] famously proved that every planar graph is 5-choosable, and Dvořák and Postle [11] observed that the DP-chromatic number of every planar graph is at most 5. Also, Molloy [24] recently improved a theorem of Johansson by showing that every triangle-free graph $G$ with maximum degree $\Delta(G)$ satisfies $\chi_{\ell}(G) \leqslant$ $(1+o(1)) \Delta(G) / \log (\Delta(G))$. Bernshteyn [5] subsequently showed that this bound also holds for the DP-chromatic number. On the other hand, Bernshteyn [4] showed that if the average degree of a graph $G$ is $d$, then $\chi_{D P}(G)=\Omega(d / \log (d))$. This is in striking contrast to the celebrated result of Alon [1] that says $\chi_{\ell}(G)=\Omega(\log (d))$. It was also recently shown in [7] that there exist planar bipartite graphs with DP-chromatic number 4 even though the list chromatic number of any planar bipartite graph is at most 3 [2]. A famous result of Galvin [13] says that if $G$ is a bipartite multigraph and $L(G)$ is the line graph of $G$, then $\chi_{\ell}(L(G))=\chi(L(G))=\Delta(G)$. However, it is also shown in [7] that every $d$-regular graph $G$ satisfies $\chi_{D P}(L(G)) \geqslant d+1$.

\subsection{Counting Proper Colorings, List Colorings, and DP-Colorings}

In 1912 Birkhoff introduced the notion of the chromatic polynomial in hopes of using it to make progress on the four color problem. For $m \in \mathbb{N}$, the chromatic polynomial of a graph $G, P(G, m)$, is the number of proper $m$-colorings of $G$. It can be shown that $P(G, m)$ is a polynomial in $m$ of degree $|V(G)|$ (see [9]). For example, $P\left(K_{n}, m\right)=\prod_{i=0}^{n-1}(m-i)$, $P\left(C_{n}, m\right)=(m-1)^{n}+(-1)^{n}(m-1), P(T, m)=m(m-1)^{n-1}$ whenever $T$ is a tree on $n$ vertices, and $P\left(K_{1} \vee G, m\right)=m P(G, m-1)$ (see [31]).

The notion of chromatic polynomial was extended to list coloring in the 1990s [21]. In particular, if $L$ is a list assignment for $G$, we use $P(G, L)$ to denote the number of proper $L$-colorings of $G$. The list color function $P_{\ell}(G, m)$ is the minimum value of $P(G, L)$ where the minimum is taken over all possible $m$-assignments $L$ for $G$. It is clear that $P_{\ell}(G, m) \leqslant P(G, m)$ for each $m \in \mathbb{N}$ since we must consider the $m$-assignment that assigns the same $m$ colors to all the vertices in $G$ when considering all possible $m$ assignments for $G$. In general, the list color function can differ significantly from the chromatic polynomial for small values of $m$. However, for large values of $m$, Wang, Qian, and Yan [30] (improving upon results in [10] and [28]) showed the following in 2017.

Theorem 1 ([30]). If $G$ is a connected graph with $l$ edges, then $P_{\ell}(G, m)=P(G, m)$ whenever $m>\frac{l-1}{\ln (1+\sqrt{2})}$.

It is also known that $P_{\ell}(G, m)=P(G, m)$ for all $m \in \mathbb{N}$ when $G$ is a cycle or chordal (see [20] and [21]). Moreover, if $P_{\ell}(G, m)=P(G, m)$ for all $m \in \mathbb{N}$, then $P_{\ell}\left(K_{n} \vee G, m\right)=$ 
$P\left(K_{n} \vee G, m\right)$ for each $n, m \in \mathbb{N}$ (see [15]). See [28] for a survey of known results and open questions on the list color function.

In 2019, Kaul and the first author introduced a DP-coloring analogue of the chromatic polynomial in hopes of gaining a better understanding of DP-coloring and using it as a tool for making progress on some open questions related to the list color function [16]. Specifically, suppose $\mathcal{H}=(L, H)$ is a cover of graph $G$. Let $P_{D P}(G, \mathcal{H})$ be the number of $\mathcal{H}$-colorings of $G$. Then, the $D P$ color function of $G, P_{D P}(G, m)$, is the minimum value of $P_{D P}(G, \mathcal{H})$ where the minimum is taken over all possible $m$-fold covers $\mathcal{H}$ of $G .{ }^{2}$ It is easy to show that for any graph $G$ and $m \in \mathbb{N}, P_{D P}(G, m) \leqslant P_{\ell}(G, m) \leqslant P(G, m) .{ }^{3}$ Note that if $G$ is a disconnected graph with components: $H_{1}, H_{2}, \ldots, H_{t}$, then $P_{D P}(G, m)=$ $\prod_{i=1}^{t} P_{D P}\left(H_{i}, m\right)$. So, we will only consider connected graphs from this point forward unless otherwise noted.

As with list coloring and DP-coloring, the list color function and DP color function of certain graphs behave similarly. However, for some graphs there are surprising differences. For example, similar to the list color function, $P_{D P}(G, m)=P(G, m)$ for every $m \in \mathbb{N}$ whenever $G$ is chordal or an odd cycle [16]. On the other hand, we have the following two results.

Theorem 2 ([16]). If $G$ is a graph with girth that is even, then there is an $N \in \mathbb{N}$ such that $P_{D P}(G, m)<P(G, m)$ whenever $m \geqslant N$. Furthermore, for any integer $g \geqslant 3$ there exists a graph $H$ with girth $g$ and an $N \in \mathbb{N}$ such that $P_{D P}(H, m)<P(H, m)$ whenever $m \geqslant N$.

This result is particularly surprising since Theorem 1 implies that the list color function of any graph eventually equals its chromatic polynomial. The following is also known.

Theorem 3 ([16]). For any graph $G$ with $n$ vertices,

$$
P(G, m)-P_{D P}(G, m)=O\left(m^{n-2}\right) \text { as } m \rightarrow \infty .
$$

In studying the tightness of Theorem 3, the authors of [16] mentioned that if $G$ is a unicyclic graph ${ }^{4}$ on $n$ vertices that contains a cycle of length 4 , then $P(G, m)-$ $P_{D P}(G, m)=\Theta\left(m^{n-3}\right)$. However, they stated that "we do not have an example of a graph $G$ such that $P(G, m)-P_{D P}(G, m)=\Theta\left(m^{n-2}\right)$." Motivated by a result of Bernshteyn, Kostochka, and Zhu [8] that says for any graph $G$ there exists an $N \leqslant 3|E(G)|$ such that $\chi_{D P}\left(K_{p} \vee G\right)=\chi\left(K_{p} \vee G\right)$ whenever $p \geqslant N$, the authors of [16] also studied $P_{D P}\left(K_{p} \vee\right.$ $G, m)$. Interestingly, it turns out that the question of whether there exist $p, N \in \mathbb{N}$ such that $P_{D P}\left(K_{p} \vee G, m\right)=P\left(K_{p} \vee G, m\right)$ whenever $m \geqslant N$ is related to the asymptotics of $P(G, m)-P_{D P}(G, m)$. In fact, the following two questions were both posed in [16]. These two questions are the focus of this note.

\footnotetext{
${ }^{2}$ We take $\mathbb{N}$ to be the domain of the DP color function of any graph.

${ }^{3}$ To prove this, recall that for any $m$-assignment $L$ for $G$, an $m$-fold cover $\mathcal{H}^{\prime}$ of $G$ such that $G$ has an $\mathcal{H}^{\prime}$-coloring if and only if $G$ has a proper $L$-coloring is constructed in [7]. It is easy to see from the construction in [7] that there is a bijection between the proper $L$-colorings of $G$ and the $\mathcal{H}^{\prime}$-colorings of $G$.

${ }^{4} \mathrm{~A}$ unicyclic graph is a connected graph containing exactly one cycle.
} 
Question 4. For any graph $G$ with $n$ vertices, is it the case that $P(G, m)-P_{D P}(G, m)=$ $O\left(m^{n-3}\right)$ as $m \rightarrow \infty$ ?

Question 5. For every graph $G$, does there exist $p, N \in \mathbb{N}$ such that $P_{D P}\left(G \vee K_{p}, m\right)=$ $P\left(G \vee K_{p}, m\right)$ whenever $m \geqslant N$ ?

In [16] it is shown that if the the answer to Question 4 is yes, then the answer to Question 5 must be yes. We will show that the answer to Question 4 is yes. This of course implies that the answer to Question 5 is yes, but we will show that its answer is yes even when $p$ is fixed to 1 .

\subsection{Summary of Results}

We begin by showing the following.

Theorem 6. Suppose $g$ is an odd integer with $g \geqslant 3$. If $G$ is a graph on $n$ vertices with girth $g$ or $g+1$, then $P(G, m)-P_{D P}(G, m)=O\left(m^{n-g}\right)$ as $m \rightarrow \infty$. Consequently, $P(M, m)-P_{D P}(M, m)=O\left(m^{|V(M)|-3}\right)$ as $m \rightarrow \infty$ for any graph $M$.

When considering the third sentence of Theorem 6 , recall that if the girth of a graph is infinite, then the graph is acyclic and therefore chordal which means the DP color function of the graph is always equal to its chromatic polynomial. A result in [16] implies that if $G$ is a unicyclic graph on $n$ vertices with girth $2 k+2$ where $k \in \mathbb{N}$, then $P(G, m)-P_{D P}(G, m)=$ $(m-1)^{n}+(m-1)^{n-2 k-1}-\left[(m-1)^{n}-(m-1)^{n-2 k-2}\right]=\Theta\left(m^{n-2 k-1}\right)$. This together with a result in [3] implies that for any odd integer $g$ with $g \geqslant 3$, if $G$ consists of a cycle on $g$ vertices and a cycle on $g+1$ vertices such that the cycles share exactly one vertex, then $P(G, m)-P_{D P}(G, m)=P\left(C_{g+1}, m\right) P\left(C_{g}, m\right) / m-P_{D P}\left(C_{g+1}, m\right) P_{D P}\left(C_{g}, m\right) / m=$ $P\left(C_{g}, m\right)\left[P\left(C_{g+1}, m\right)-P_{D P}\left(C_{g+1}, m\right)\right] / m=P\left(C_{g}, m\right)=\Theta\left(m^{g}\right)$. This demonstrates the tightness of Theorem 6 for all possible girths.

We end this note by proving the following.

Theorem 7. For any graph $G$, there is an $N \in \mathbb{N}$ such that $P_{D P}\left(K_{1} \vee G, m\right)=P\left(K_{1} \vee\right.$ $G, m)$ whenever $m \geqslant N$.

Theorem 7 shows that the DP color function of $K_{1} \vee G$ behaves like the list color function of $K_{1} \vee G$ since the DP color function of $K_{1} \vee G$ eventually equals the chromatic polynomial of $K_{1} \vee G$. It is worth mentioning that in this note no attempt has been made to minimize the value of $N$ in Theorem 7 . It would be interesting to study the threshold at which $P_{D P}\left(K_{1} \vee G, m\right)=P\left(K_{1} \vee G, m\right)$ for a given graph $G$.

\section{Proofs of Results}

The key to proving our results is generalizing the proof technique of the following classical result to the context of DP-coloring. 
Theorem 8 ([32]). Suppose $G$ is a graph. Then,

$$
P(G, m)=\sum_{A \subseteq E(G)}(-1)^{|A|} m^{k_{A}}
$$

where $k_{A}$ is the number of components of the spanning subgraph of $G$ with edge set $A$.

The next four results will also be useful tools to keep in mind.

Proposition 9 ([32]). Suppose $G$ is a graph on $n$ vertices. Then there are nonnegative integers $a_{0}, \ldots, a_{n}$ such that $P(G, m)=\sum_{i=0}^{n}(-1)^{i} a_{i} m^{n-i}$. Furthermore, if $G$ has $c$ components, then $a_{0}, \ldots, a_{n-c}$ are all positive integers, and $a_{n-c+1}=\cdots=a_{n}=0$.

Proposition 10 ([32]). Suppose $G$ is a graph with $s$ edges and $n$ vertices having girth $g \in \mathbb{N}$. Suppose $P(G, m)=\sum_{i=0}^{n}(-1)^{i} a_{i} m^{n-i}$. Then, for $i=0,1, \ldots, g-2$

$$
a_{i}=\left(\begin{array}{l}
s \\
i
\end{array}\right) \text { and } a_{g-1}=\left(\begin{array}{c}
s \\
g-1
\end{array}\right)-t
$$

where $t$ is the number of cycles of length $g$ contained in $G$.

Proposition 11 ([16]). Suppose $T$ is a tree and $\mathcal{H}=(L, H)$ is an $m$-fold cover of $T$ such that $E_{H}(L(u), L(v))$ is a perfect matching whenever uv $\in E(T)$. Then, $\mathcal{H}$ has a canonical labeling.

Proposition 12. Suppose that $\mathcal{H}=(L, H)$ is an m-fold cover of graph $G$ and $\mathcal{H}$ has a canonical labeling. Let $B_{i}=\{(v, i): v \in V(G)\}$ for each $i \in[m]$. Then, $I \subset V(H)$ satisfies: $|I \cap L(v)|=1$ for each $v \in V(G)$ and $H[I]$ is isomorphic to $G$ if and only if $I=B_{j}$ for some $j \in[m]$.

Proof. For each $i \in[m]$, it is clear that $\left|B_{i} \cap L(v)\right|=1$ for each $v \in V(G)$ and $H\left[B_{i}\right]$ is isomorphic to $G$. Conversely, suppose that $I \notin\left\{B_{1}, \ldots, B_{m}\right\}$. Since $|I \cap L(v)|=1$ for each $v \in V(G), H[I]$ has fewer edges than $G$ contradicting the fact that $H[I]$ is isomorphic to $G$.

\section{$2.1 \quad$ Proof of Theorem 6}

We will now introduce some notation that will be used for the remainder of this note. Suppose that $G$ is a graph on $n \geqslant 3$ vertices with $|E(G)| \geqslant 3$. Let $s=|E(G)|$, and $E(G)=\left\{e_{1}, \ldots, e_{s}\right\}$. Also, for some $m \in \mathbb{N}$ suppose that $\mathcal{H}=(L, H)$ is an $m$-fold cover of $G$ satisfying $\left|E_{H}(L(u), L(v))\right|=m$ whenever $u v \in E(G)$.

Let $\mathcal{U}=\{I \subseteq V(H):|L(v) \cap I|=1$ for each $v \in V(G)\}$. Clearly, $|\mathcal{U}|=m^{n}$. Now, for each $i \in[s]$, suppose $e_{i}=u_{i} v_{i}$, and let $S_{i}$ be the set consisting of each $I \in \mathcal{U}$ with the property that $H[I]$ contains an edge in $E_{H}\left(L\left(u_{i}\right), L\left(v_{i}\right)\right)$. Also, for each $i \in[s]$ let $C_{i}=\mathcal{U}-S_{i}$. Clearly,

$$
P_{D P}(G, \mathcal{H})=\left|\bigcap_{i=1}^{s} C_{i}\right| .
$$


So, by the Inclusion-Exclusion Principle, we see that

$$
P_{D P}(G, \mathcal{H})=|\mathcal{U}|-\left|\bigcup_{i=1}^{s} S_{i}\right|=m^{n}-\sum_{k=1}^{s}(-1)^{k-1}\left(\sum_{1 \leqslant i_{1}<\cdots<i_{k} \leqslant s}\left|\bigcap_{j=1}^{k} S_{i_{j}}\right|\right) .
$$

The following Lemma is the key to our proof of Theorem 6 .

Lemma 13. Assuming the set-up established above, suppose that $G$ is a graph of girth $g \in \mathbb{N}$. Then, the following three statements hold.

(i) For any $k \in[g-1]$ and $i_{1}, \ldots, i_{k} \in[s]$ satisfying $i_{1}<\cdots<i_{k},\left|\bigcap_{j=1}^{k} S_{i_{j}}\right|=m^{n-k}$.

(ii) If $e_{i_{1}}, \ldots, e_{i_{g}}$ are distinct edges in $G$, then $\left|\bigcap_{j=1}^{g} S_{i_{j}}\right| \leqslant m^{n-g+1}$. Moreover,

$\left|\bigcap_{j=1}^{g} S_{i_{j}}\right|=m^{n-g}$ when $e_{i_{1}}, \ldots, e_{i_{g}}$ are not the edges of a g-cycle in $G$.

(iii) For any $k \geqslant g+1$ and $i_{1}, \ldots, i_{k} \in[s]$ satisfying $i_{1}<\cdots<i_{k},\left|\bigcap_{j=1}^{k} S_{i_{j}}\right| \leqslant m^{n-g}$.

Proof. For Statement (i), suppose that $G^{\prime}$ is the spanning subgraph of $G$ with $E\left(G^{\prime}\right)=$ $\left\{e_{i_{1}}, \ldots, e_{i_{k}}\right\}$. Let $H^{\prime}=H-\bigcup_{x y \in E(G)-E\left(G^{\prime}\right)} E_{H}(L(x), L(y))$. Since $G$ has girth $g$ and $k \in[g-1], G^{\prime}$ is an acyclic graph with $n-k$ components. Suppose the components of $G^{\prime}$ are $W_{1}, \ldots, W_{n-k}$ (each component is a tree). Note that we can construct each element $I$ of $\bigcap_{j=1}^{k} S_{i_{j}}$ in $(n-k)$ steps as follows. For each $i \in[n-k]$ consider the component $W_{i}$. Suppose $V\left(W_{i}\right)=\left\{w_{1}, \ldots, w_{l}\right\}$. Choose one element from each of $L\left(w_{1}\right), \ldots, L\left(w_{l}\right)$ so that the subgraph of $H^{\prime}$ induced by the set containing these chosen elements is isomorphic to $W_{i}$. Then, place these chosen elements in $I$. By Propositions 11 and 12, this step can be done in $m$ ways (regardless of the choices made in previous steps). So, $\left|\bigcap_{j=1}^{k} S_{i_{j}}\right|=m^{n-k}$.

For Statement (ii), the first part follows from Statement (i) since $\left|\bigcap_{j=1}^{g} S_{i_{j}}\right| \leqslant\left|\bigcap_{j=1}^{g-1} S_{i_{j}}\right|=m^{n-g+1}$. So, suppose that $e_{i_{1}}, \ldots, e_{i_{g}}$ are not the edges of a $g$-cycle in $G$. Let $G^{\prime \prime}$ be the spanning subgraph of $G$ with $E\left(G^{\prime \prime}\right)=\left\{e_{i_{1}}, \ldots, e_{i_{g}}\right\}$. Clearly, $G^{\prime \prime}$ is an acyclic graph with $n-g$ components. We can obtain $\left|\bigcap_{j=1}^{g} S_{i_{j}}\right|=m^{n-g}$ by using an argument similar to the argument used for the proof of Statement (i).

For Statement (iii), notice that we can assume without loss of generality that $e_{i_{1}}, \ldots, e_{i_{g}}$ are not the edges of a $g$-cycle in $G$. So, by Statement (ii), we see that $\left|\bigcap_{j=1}^{k} S_{i_{j}}\right| \leqslant\left|\bigcap_{j=1}^{g} S_{i_{j}}\right|=m^{n-g}$.

We are now ready to prove Theorem 6 .

Proof. Suppose $s=|E(G)|$ and $t$ is the number of $g$-cycles in $G$ (note that $t=0$ in the case that $G$ has girth $g+1)$. Since $g$ is odd, Propositions 9 and 10 tell us that there is an $N \in \mathbb{N}$ such that

$$
P(G, m) \leqslant\left(\left(\begin{array}{c}
s \\
g-1
\end{array}\right)-t\right) m^{n-g+1}+\sum_{i=0}^{g-2}(-1)^{i}\left(\begin{array}{l}
s \\
i
\end{array}\right) m^{n-i}
$$


whenever $m \geqslant N$. Suppose that $m$ is a fixed natural number satisfying $m \geqslant N$.

Suppose that $\mathcal{H}=(L, H)$ is an $m$-fold cover of $G$ satisfying $P_{D P}(G, \mathcal{H})=P_{D P}(G, m)$. Clearly, we may assume that $\left|E_{H}(L(u), L(v))\right|=m$ whenever $u v \in E(G)$. Now, assume we use the same notation described at the start of this Subsection. By Statement (i) of Lemma 13, we have that

$$
\begin{aligned}
& P_{D P}(G, m) \\
& =m^{n}+\sum_{k=1}^{s}(-1)^{k}\left(\sum_{1 \leqslant i_{1}<\cdots<i_{k} \leqslant s}\left|\bigcap_{j=1}^{k} S_{i_{j}}\right|\right) \\
& =\sum_{i=0}^{g-1}(-1)^{i}\left(\begin{array}{c}
s \\
i
\end{array}\right) m^{n-i}-\sum_{1 \leqslant i_{1}<\cdots<i_{g} \leqslant s}\left|\bigcap_{j=1}^{g} S_{i_{j}}\right|+\sum_{k=g+1}^{s}(-1)^{k}\left(\sum_{1 \leqslant i_{1}<\cdots<i_{k} \leqslant s}\left|\bigcap_{j=1}^{k} S_{i_{j}}\right|\right) .
\end{aligned}
$$

We see that Statement (ii) of Lemma 13 implies that

$$
\sum_{1 \leqslant i_{1}<\cdots<i_{g} \leqslant s}\left|\bigcap_{j=1}^{g} S_{i_{j}}\right| \leqslant t m^{n-g+1}+\left(\left(\begin{array}{l}
s \\
g
\end{array}\right)-t\right) m^{n-g} .
$$

Furthermore, Statement (iii) of Lemma 13 implies that

$$
\sum_{k=g+1}^{s}(-1)^{k}\left(\sum_{1 \leqslant i_{1}<\cdots<i_{k} \leqslant s}\left|\bigcap_{j=1}^{k} S_{i_{j}}\right|\right) \geqslant-2^{s} m^{n-g}
$$

These facts imply that

$$
P_{D P}(G, m) \geqslant\left(\left(\begin{array}{c}
s \\
g-1
\end{array}\right)-t\right) m^{n-g+1}+\sum_{i=0}^{g-2}(-1)^{i}\left(\begin{array}{l}
s \\
i
\end{array}\right) m^{n-i}-\left(\left(\begin{array}{l}
s \\
g
\end{array}\right)-t\right) m^{n-g}-2^{s} m^{n-g} .
$$

So, we see that

$$
P(G, m)-P_{D P}(G, m) \leqslant\left(\left(\begin{array}{l}
s \\
g
\end{array}\right)-t+2^{s}\right) m^{n-g} .
$$

The desired result immediately follows.

\subsection{Proof of Theorem 7}

Notice that the result of Theorem 7 is obvious when $G$ is acyclic since the cone of such a graph is chordal. So, throughout this Subsection suppose that $G$ is a graph with $n-1$ vertices where $n \geqslant 4$ and $s \geqslant 3$ edges. Suppose that $E(G)=\left\{e_{1}, \ldots, e_{s}\right\}$. Also, suppose that $M=K_{1} \vee G$, and $w$ is the vertex corresponding to the copy of $K_{1}$ used to form $M$. We use $e_{s+1}, \ldots, e_{s+n-1}$ to denote the edges in $E(M)$ that have $w$ as an endpoint. We want to show that $P_{D P}(M, m)=P(M, m)$ for sufficiently large $m$, or equivalently, $P_{D P}(M, m) \geqslant P(M, m)$ for sufficiently large $m$. 
Since $M$ is a graph with $n$ vertices and $n+s-1$ edges, Propositions 9 and 10 tell us that

$P(M, m)=m^{n}-(n+s-1) m^{n-1}+\left(\left(\begin{array}{c}n+s-1 \\ 2\end{array}\right)-t\right) m^{n-2}-a_{3} m^{n-3}+O\left(m^{n-4}\right)$ as $m \rightarrow \infty$

where $t$ is the number of 3 -cycles contained in $M$ (note that $t \geqslant s$ ). We now give a formula for $a_{3}$. Let $A=\left\{A_{1}, \ldots, A_{q}\right\}$ be the set of spanning subgraphs of $M$ with $(n-3)$ components. For each $i \in[q]$, it is straightforward to verify that $3 \leqslant\left|E\left(A_{i}\right)\right| \leqslant 6$. For $i \in\{3,4,5,6\}$, let $P_{i}=\left\{E\left(A_{j}\right): A_{j} \in A,\left|E\left(A_{j}\right)\right|=i\right\}$. By Theorem $8, a_{3}=$ $\left|P_{3}\right|-\left|P_{4}\right|+\left|P_{5}\right|-\left|P_{6}\right|$.

In this Subsection we are interested in finding a lower bound for $P_{D P}(M, m)$. So, whenever $\mathcal{H}=(L, H)$ is an $m$-fold cover for $M$, we will assume that $\left|E_{H}(L(u), L(v))\right|=m$ for each $u v \in E(M)$. We will also suppose without loss of generality that $L(u)=\{(u, j)$ : $j \in[m]\}$ for each $u \in V(M)$, and $(w, j)(v, j) \in E(H)$ for each $v \in V(G)$ and $j \in[m]$ (this is permissible by Proposition 11 since the spanning subgraph of $M$ with edge set $\left\{e_{s+1}, \ldots, e_{s+n-1}\right\}$ is a tree).

Suppose $\mathcal{H}=(L, H)$ is an $m$-fold cover for $M$. For each $e_{i} \in E(G)$ we suppose that $e_{i}=u_{i} v_{i}$, and we let $x_{i, \mathcal{H}}{ }^{5}$ be the number of edges in $E_{H}\left(L\left(u_{i}\right), L\left(v_{i}\right)\right)$ that connect endpoints with differing second coordinates. Finally, we let $x_{\mathcal{H}}=\sum_{i=1}^{s} x_{i, \mathcal{H}}$. Clearly, if $x_{\mathcal{H}}=0$, then $\mathcal{H}$ has a canonical labeling and $P_{D P}(M, \mathcal{H})=P(M, m)$. Also, $x_{\mathcal{H}}$ is the number of cross edges in $H$ that connect vertices with differing second coordinates.

For the next Lemma assume that $\mathcal{H}=(L, H)$ is an $m$-fold cover for $M$, and assume we are using the same notation as the beginning of Subsection 2.1 (with $M$ playing the role of $G)$.

Lemma 14. The following statments hold.

(i) $\sum_{1 \leqslant i_{1}<\cdots<i_{3} \leqslant n+s-1}\left|\bigcap_{j=1}^{3} S_{i_{j}}\right| \leqslant t m^{n-2}-x_{\mathcal{H}} m^{n-3}+\left|P_{3}\right| m^{n-3}$,

(ii) $\sum_{1 \leqslant i_{1}<\cdots<i_{4} \leqslant n+s-1}\left|\bigcap_{j=1}^{4} S_{i_{j}}\right| \geqslant\left|P_{4}\right| m^{n-3}-2\left|P_{4}\right| x_{\mathcal{H}} m^{n-4}$,

(iii) $\sum_{1 \leqslant i_{1}<\cdots<i_{5} \leqslant n+s-1}\left|\bigcap_{j=1}^{5} S_{i_{j}}\right| \leqslant\left|P_{5}\right| m^{n-3}+\left(\left(\begin{array}{c}n+s-1 \\ 5\end{array}\right)-\left|P_{5}\right|\right) m^{n-4}$,

(iv) $\sum_{1 \leqslant i_{1}<\cdots<i_{6} \leqslant n+s-1}\left|\bigcap_{j=1}^{6} S_{i_{j}}\right| \geqslant\left|P_{6}\right| m^{n-3}-2\left|P_{6}\right| x_{\mathcal{H}} m^{n-4}$, and

(v) For $k \geqslant 7, \sum_{1 \leqslant i_{1}<\cdots<i_{k} \leqslant n+s-1}\left|\bigcap_{j=1}^{k} S_{i_{j}}\right| \leqslant\left(\begin{array}{c}n+s-1 \\ k\end{array}\right) m^{n-4}$.

Proof. For Statement (i), suppose $x, y$, and $z$ are distinct edges in $E(M)$. Let $M^{\prime}$ be the spanning subgraph of $M$ with $E\left(M^{\prime}\right)=\{x, y, z\}$. If $x, y$, and $z$ form a 3-cycle in $M$ containing $w$, then $M^{\prime}$ consists of this 3-cycle and $n-3$ isolated vertices. Notice that each 3-cycle in $M$ containing $w$ contains exactly one edge in $E(G)$. So, we suppose that $z=e_{i}$ for some $i \in[s]$, then it is clear that $\left|S_{x} \cap S_{y} \cap S_{z}\right|=m^{n-3}\left(m-x_{i}\right)=m^{n-2}-x_{i} m^{n-3}$. In the case that $x, y$, and $z$ form a 3-cycle in $M$ not containing $w$, then Lemma 13 implies that $\left|S_{x} \cap S_{y} \cap S_{z}\right| \leqslant m^{n-2}$. Finally, in the case that $x, y$, and $z$ do not form a 3 -cycle in $M$

\footnotetext{
${ }^{5}$ We will just write $x_{i}$ when $\mathcal{H}$ is clear from context.
} 
(note that there are $\left|P_{3}\right|$ such sets of three edges), Lemma 13 implies $\left|S_{x} \cap S_{y} \cap S_{z}\right|=m^{n-3}$. Statement (i) now follows immediately from these facts.

For Statement (ii), suppose $a, x, y$, and $z$ are distinct edges in $E(M)$. Let $M^{\prime}$ be the spanning subgraph of $M$ with $E\left(M^{\prime}\right)=\{a, x, y, z\}$. Let

$H^{\prime}=H-\bigcup_{x y \in E(M)-E\left(M^{\prime}\right)} E_{H}(L(x), L(y))$. If $M^{\prime}$ contains a cycle, then $M^{\prime}$ contains one cycle and consists of $n-3$ components (note that there are $\left|P_{4}\right|$ sets of four edges for which this happens). Suppose that the components of $M^{\prime}$ are $W_{1}, \ldots, W_{n-3}$, and assume that $W_{1}$ is the component of $M^{\prime}$ containing the cycle. Also, suppose that $V\left(W_{1}\right)=\left\{w_{1}, \ldots, w_{l}\right\}$. Now, let $B_{i}=\left\{\left(w_{j}, i\right): j \in[l]\right\}$ for each $i \in[m]$. If $H^{\prime}\left[B_{i}\right]$ is not isomorphic to $W_{1}$, then an element of $B_{i}$ must be the endpoint of a cross edge in $H$ that connects vertices with differing second coordinates. Let $\mathcal{B}$ consist of each $B_{i} \in\left\{B_{1}, \ldots, B_{m}\right\}$ with the property that $H^{\prime}\left[B_{i}\right]$ is not isomorphic to $W_{1}$. Notice this means that for each $B_{j} \in$ $\left\{B_{1}, \ldots, B_{m}\right\}-\mathcal{B}, H^{\prime}\left[B_{j}\right]$ is isomorphic to $W_{1}$, and there are are least $\left|\left\{B_{1}, \ldots, B_{m}\right\}-\mathcal{B}\right|$ ways to select one element from each of $L\left(w_{1}\right), \ldots, L\left(w_{l}\right)$ so that the subgraph of $H^{\prime}$ induced by the set containing these chosen elements is isomorphic to $W_{1}$. Let $\mathcal{E}$ be the set of cross edges in $H$ that connect vertices with differing second coordinates (note that $\left.|\mathcal{E}|=x_{\mathcal{H}}\right)$. We can construct a function $\eta: \mathcal{B} \rightarrow \mathcal{E}$ that maps each $B_{i} \in \mathcal{B}$ to one of the edges in $\mathcal{E}$ that has an endpoint in $B_{i}$. Furthermore, if $B_{i}, B_{j}$, and $B_{t}$ are distinct elements of $\mathcal{B}$, then it is not possible for $\eta\left(B_{i}\right)=\eta\left(B_{j}\right)=\eta\left(B_{t}\right)$ since an edge only has two endpoints. Consequently, $\left|\left\{B_{1}, \ldots, B_{m}\right\}-\mathcal{B}\right| \geqslant\left(m-2 x_{\mathcal{H}}\right)$. So, $\left|S_{a} \cap S_{x} \cap S_{y} \cap S_{z}\right| \geqslant m^{n-4}\left(m-2 x_{\mathcal{H}}\right)=m^{n-3}-2 x_{\mathcal{H}} m^{n-4}$. Statement (ii) now immediately follows.

For Statement (iii), suppose $a, b, x, y$, and $z$ are distinct edges in $E(M)$. Let $M^{\prime}$ be the spanning subgraph of $M$ with $E\left(M^{\prime}\right)=\{a, b, x, y, z\}$. Let

$H^{\prime}=H-\bigcup_{x y \in E(M)-E\left(M^{\prime}\right)} E_{H}(L(x), L(y))$. Suppose $M^{\prime}$ consists of $n-3$ components (note that there are $\left|P_{5}\right|$ sets of five edges for which this happens). Suppose that the components of $M^{\prime}$ are $W_{1}, \ldots, W_{n-3}$. Note that we can construct each element $I$ of $\left(S_{a} \cap S_{b} \cap S_{x} \cap S_{y} \cap S_{z}\right)$ in $(n-3)$ steps as follows. For each $i \in[n-3]$ consider the component $W_{i}$. If $\{a, b, x, y, z\} \cap E\left(W_{i}\right) \neq \emptyset$, then $V\left(W_{i}\right)$ has at least 2 elements, say $V\left(W_{i}\right)=\left\{w_{1}, \ldots, w_{l}\right\}$, choose one element from each of $L\left(w_{1}\right), \ldots, L\left(w_{l}\right)$ so that the subgraph of $H^{\prime}$ induced by these chosen elements is isomorphic to $W_{i}$ (this can be done in at most $m$ ways $\left.{ }^{6}\right)$. Then, place these chosen elements in $I$. If $\{a, b, x, y, z\} \cap E\left(W_{i}\right)=\emptyset$, then $W_{i}$ is a single vertex, say $V\left(W_{i}\right)=\{q\}$, and we choose an element of $L(q)$ to place in $I$. Notice that in either case there are at most $m$ ways to complete the step. Consequently, $\left|S_{a} \cap S_{b} \cap S_{x} \cap S_{y} \cap S_{z}\right| \leqslant m^{n-3}$. A similar argument shows that when $M^{\prime}$ has fewer than $n-3$ components, $\left|S_{a} \cap S_{b} \cap S_{x} \cap S_{y} \cap S_{z}\right| \leqslant m^{n-4}$. Statement (iii) now follows from the fact that $\sum_{1 \leqslant i_{1}<\cdots<i_{5} \leqslant n+s-1}\left|\bigcap_{j=1}^{5} S_{i_{j}}\right|$ has $\left(\begin{array}{c}n+s-1 \\ 5\end{array}\right)$ terms.

For Statement (iv), suppose $a, b, c, x, y$, and $z$ are distinct edges in $E(M)$. Let $M^{\prime}$ be the spanning subgraph of $M$ with $E\left(M^{\prime}\right)=\{a, b, c, x, y, z\}$. Suppose $M^{\prime}$ consists of $n-3$ components (note that there are $\left|P_{6}\right|$ sets of six edges for which this happens). It is easy to see that $M^{\prime}$ must consist of a complete graph on four vertices and $n-4$

\footnotetext{
${ }^{6}$ To see why this is so, consider a spanning tree of $W_{i}$ and apply Propositions 11 and 12.
} 
isolated vertices. Suppose that the components of $M^{\prime}$ are $W_{1}, \ldots, W_{n-3}$, and assume that $W_{1}=K_{4}$. Using an argument similar to the argument used to prove Statement (ii), we obtain $\left|S_{a} \cap S_{b} \cap S_{c} \cap S_{x} \cap S_{y} \cap S_{z}\right| \geqslant m^{n-4}\left(m-2 x_{\mathcal{H}}\right)=m^{n-3}-2 x_{\mathcal{H}} m^{n-4}$. Statement (iv) now immediately follows.

For Statement $(\mathrm{v})$, suppose $k \geqslant 7$ and $1 \leqslant i_{1}<\cdots<i_{k} \leqslant n+s-1$. Let $M^{\prime}$ be the spanning subgraph of $M$ with $E\left(M^{\prime}\right)=\left\{e_{i_{1}}, \ldots, e_{i_{k}}\right\}$. Then, $M^{\prime}$ must consist of at most $n-4$ components. An argument similar to the argument used to prove Statement (iii) then yields $\left|\bigcap_{j=1}^{k} S_{i_{j}}\right| \leqslant m^{n-4}$. Statement (v) now immediately follows.

We need one more Lemma before proving Theorem 7 .

Lemma 15. Suppose that $m \geqslant 2\left(\left|P_{4}\right|+\left|P_{6}\right|\right)$, and $\mathcal{H}=(L, H)$ is an $m$-fold cover for $M$ with $x_{\mathcal{H}}>0$. Then,

$$
\begin{aligned}
& P_{D P}(M, \mathcal{H}) \\
& \geqslant m^{n}-(n+s-1) m^{n-1}+\left(\left(\begin{array}{c}
n+s-1 \\
2
\end{array}\right)-t\right) m^{n-2}-a_{3} m^{n-3} \\
& +m^{n-3}-\left(2\left(\left|P_{4}\right|+\left|P_{6}\right|+2^{n+s-2}\right)\right) m^{n-4} .
\end{aligned}
$$

Proof. Using the notation established in Subsection 2.1 (with $M$ playing the role of $G$ ) along with Lemma 13, we know that

$$
\begin{aligned}
& P_{D P}(M, \mathcal{H}) \\
& =m^{n}+\sum_{k=1}^{n+s-1}(-1)^{k}\left(\sum_{1 \leqslant i_{1}<\cdots<i_{k} \leqslant n+s-1}\left|\bigcap_{j=1}^{k} S_{i_{j}}\right|\right) \\
& =m^{n}-(n+s-1) m^{n-1}+\left(\begin{array}{c}
n+s-1 \\
2
\end{array}\right) m^{n-2}-\sum_{1 \leqslant i_{1}<\cdots<i_{3} \leqslant n+s-1}\left|\bigcap_{j=1}^{3} S_{i_{j}}\right| \\
& +\sum_{1 \leqslant i_{1}<\cdots<i_{4} \leqslant n+s-1}\left|\bigcap_{j=1}^{4} S_{i_{j}}\right|-\sum_{1 \leqslant i_{1}<\cdots<i_{5} \leqslant n+s-1}^{5}\left|\bigcap_{j=1}^{5} S_{i_{j}}\right|+\sum_{1 \leqslant i_{1}<\cdots<i_{6} \leqslant n+s-1} S_{j=1} S_{i_{j}} \mid \\
& +\sum_{k=7}^{n+s-1}(-1)^{k}\left(\sum_{1 \leqslant i_{1}<\cdots<i_{k} \leqslant n+s-1}^{k} \mid\right)
\end{aligned}
$$

Then, Lemma 14 yields:

$$
\begin{aligned}
& P_{D P}(M, \mathcal{H}) \\
& \geqslant m^{n}-(n+s-1) m^{n-1}+\left(\begin{array}{c}
n+s-1 \\
2
\end{array}\right) m^{n-2}-\left(t m^{n-2}-x_{\mathcal{H}} m^{n-3}+\left|P_{3}\right| m^{n-3}\right) \\
& +\left|P_{4}\right| m^{n-3}-2\left|P_{4}\right| x_{\mathcal{H}} m^{n-4}-\left(\left|P_{5}\right| m^{n-3}+\left(\left(\begin{array}{c}
n+s-1 \\
5
\end{array}\right)-\left|P_{5}\right|\right) m^{n-4}\right) \\
& +\left|P_{6}\right| m^{n-3}-2\left|P_{6}\right| x_{\mathcal{H}} m^{n-4}-\sum_{k=7}^{n+s-1}\left(\begin{array}{c}
n+s-1 \\
k
\end{array}\right) m^{n-4}
\end{aligned}
$$




$$
\begin{aligned}
& \geqslant m^{n}-(n+s-1) m^{n-1}+\left(\left(\begin{array}{c}
n+s-1 \\
2
\end{array}\right)-t\right) m^{n-2}-a_{3} m^{n-3} \\
& +\left(m-2\left|P_{4}\right|-2\left|P_{6}\right|\right) x_{\mathcal{H}} m^{n-4}-2^{n+s-1} m^{n-4} \\
& \geqslant m^{n}-(n+s-1) m^{n-1}+\left(\left(\begin{array}{c}
n+s-1 \\
2
\end{array}\right)-t\right) m^{n-2}-a_{3} m^{n-3} \\
& +m^{n-3}-\left(2\left(\left|P_{4}\right|+\left|P_{6}\right|+2^{n+s-2}\right)\right) m^{n-4} .
\end{aligned}
$$

We now prove Theorem 7 .

Proof. By Propositions 9 and 10 there are $C, N_{1} \in \mathbb{N}$ such that $P(M, m) \leqslant m^{n}-(n+$ $s-1) m^{n-1}+\left(\left(\begin{array}{c}n+s-1 \\ 2\end{array}\right)-t\right) m^{n-2}-a_{3} m^{n-3}+C m^{n-4}$ whenever $m \geqslant N_{1}$. Also, when $m \geqslant 2\left(\left|P_{4}\right|+\left|P_{6}\right|\right)$ and $\mathcal{H}=(L, H)$ is an $m$-fold cover for $M$ with $x_{\mathcal{H}}>0$, Lemma 15 tells us $P_{D P}(M, \mathcal{H}) \geqslant m^{n}-(n+s-1) m^{n-1}+\left(\left(\begin{array}{c}n+s-1 \\ 2\end{array}\right)-t\right) m^{n-2}-a_{3} m^{n-3}+m^{n-3}-\left(2\left(\left|P_{4}\right|+\right.\right.$ $\left.\left.\left|P_{6}\right|+2^{n+s-2}\right)\right) m^{n-4}$. Finally, there must be an $N_{2} \in \mathbb{N}$ such that $m^{n-3}-\left(2\left(\left|P_{4}\right|+\left|P_{6}\right|+\right.\right.$ $\left.\left.2^{n+s-2}\right)+C\right) m^{n-4} \geqslant 0$ whenever $m \geqslant N_{2}$.

Let $N=\max \left\{N_{1}, N_{2}, 2\left(\left|P_{4}\right|+\left|P_{6}\right|\right)\right\}$. If $m \geqslant N$ and $\mathcal{H}=(L, H)$ is an $m$-fold cover for $M$ with $x_{\mathcal{H}}>0$, then $P_{D P}(M, \mathcal{H})-P(M, m) \geqslant m^{n-3}-\left(2\left(\left|P_{4}\right|+\left|P_{6}\right|+2^{n+s-2}\right)+\right.$ $C) m^{n-4} \geqslant 0$. Since we know that when $\mathcal{H}=(L, H)$ is an $m$-fold cover for $M$ with $x_{\mathcal{H}}=0, P_{D P}(M, \mathcal{H})=P(M, m)$, we may conclude that $P_{D P}(M, m)=P(M, m)$ whenever $m \geqslant N$.

\section{Acknowledgment}

The authors would like to thank Hemanshu Kaul and Alexandr Kostochka for their guidance and encouragement. The authors would also like to thank Michael Maxfield and the anonymous referees for their helpful comments that improved the readability of this note.

\section{References}

[1] N. Alon, Degrees and choice numbers, Random Structures 85 Algorithms 16 (2000), 364-368.

[2] N. Alon and M. Tarsi, Colorings and orientations of graphs, Combinatorica 12 (1992), $125-134$.

[3] J. Becker, J. Hewitt, H. Kaul, M. Maxfield, J. Mudrock, D. Spivey, S. Thomason, and T. Wagstrom, The DP Color Function of Joins and Vertex-Gluings of Graphs, arXiv:2104.12268, 2021.

[4] A. Bernshteyn, The asymptotic behavior of the correspondence chromatic number, Discrete Mathematics, 339 (2016), 2680-2692.

[5] A. Bernshteyn, The Johansson-Molloy Theorem for DP-coloring, Random Structures 85 Algorithms 54:4 (2019), 653-664.

[6] A. Bernshteyn and A. Kostochka, Sharp Dirac's theorem for DP-critical graphs, Journal of Graph Theory 88 (2018), 521-546. 
[7] A. Bernshteyn and A. Kostochka, On differences between DP-coloring and list coloring, Siberian Advances in Mathematics 21:2 (2018), 61-71.

[8] A. Bernshteyn, A. Kostochka, and X. Zhu, DP-colorings of graphs with high chromatic number, European Journal of Combinatorics 65 (2017), 122-129.

[9] G. D. Birkhoff, A determinant formula for the number of ways of coloring a map, The Annals of Mathematics 14 (1912), 42-46.

[10] Q. Donner, On the number of list-colorings, Journal of Graph Theory 16 (1992), 239-245.

[11] Z. Dvořák and L. Postle, Correspondence coloring and its application to list-coloring planar graphs wihtout cycles of lengths 4 to 8, Journal of Combinatorial Theory Series B 129 (2018), 38-54.

[12] P. Erdős, A. L. Rubin, and H. Taylor, Choosability in graphs, Congressus Numerantium 26 (1979), 125-127.

[13] F. Galvin, The list chromatic index of a bipartite multigraph, Journal of Combinatorial Theory Series B 63 (1995), no. 1, 153-158.

[14] J. Kahn, Asymptotically good list colorings, Journal of Combinatorial Theory Series A 73 (1996), 1-59.

[15] H. Kaul and J. Mudrock, Criticality, the list color function, and list coloring the Cartesian product of graphs, Journal of Combinatorics 12 (2021), to appear.

[16] H. Kaul and J. Mudrock, On the chromatic polynomial and counting DP-colorings of graphs, Advances in Applied Mathematics 123 (2021), article 103121.

[17] H. Kaul and J. Mudrock, Combinatorial Nullstellensatz and DP-coloring of Graphs, Discrete Mathematics 343 (2020), article 112115.

[18] S-J. Kim and K. Ozeki, A note on a Brooks' type theorem for DP-coloring, Journal of Graph Theory 91(2) (2019), 148-161.

[19] S-J. Kim and K. Ozeki, A sufficient condition for DP-4-colorability, Discrete Mathematics 341 (2018), 1983-1986.

[20] R. Kirov and R. Naimi, List coloring and $n$-monophilic graphs, Ars Combinatoria 124 (2016), 329-340.

[21] A. V. Kostochka and A. Sidorenko, Problem Session of the Prachatice Conference on Graph Theory, Fourth Czechoslovak Symposium on Combinatorics, Graphs and Complexity, Ann. Discrete Math. 51 (1992), 380.

[22] R. Liu and X. Li., Every planar graph without 4-cycles adjacent to two triangles is DP-4-colorable, Discrete Mathematics 342 (2019), 623-627.

[23] R. Liu, S. Loeb, Y. Yin, and G. Yu, DP-3-coloring of some planar graphs, Discrete Mathematics 342 (2019), 178-189.

[24] M. Molloy, The list chromatic number of graphs with small clique number, Journal of Combinatorial Theory Series B 134 (2019), 264-284. 
[25] M. Molloy, Asymptotically good edge correspondence colouring, arXiv: 1808.08594, 2018.

[26] J. Mudrock, A note on the DP-chromatic number of complete bipartite graphs, Discrete Mathematics 341 (2018), 3148-3151.

[27] C. Thomassen, Every planar graph is 5-choosable, Journal of Combinatorial Theory Series B 62 (1994), 180-181.

[28] C. Thomassen, The chromatic polynomial and list colorings, Journal of Combinatorial Theory Series B 99 (2009), 474-479.

[29] V. G. Vizing, Coloring the vertices of a graph in prescribed colors, Diskret. Analiz. no. 29, Metody Diskret. Anal. v Teorii Kodovi Skhem 101 (1976), 3-10.

[30] W. Wang, J. Qian, and Z. Yan, When does the list-coloring function of a graph equal its chromatic polynomial, Journal of Combinatorial Theory Series B 122 (2017), $543-549$.

[31] D. B. West, (2001) Introduction to Graph Theory. Upper Saddle River, NJ: Prentice Hall.

[32] H. Whitney, A logical expansion in mathematics, Bulletin of the American Mathematical Society 38 (1932), 572-579. 